\title{
Les coordinateurs, les contributeurs
}

Ce livre est un ouvrage collectif. Il est le fruit du travail d'une dizaine de personnes.

\section{Les coordinateurs}

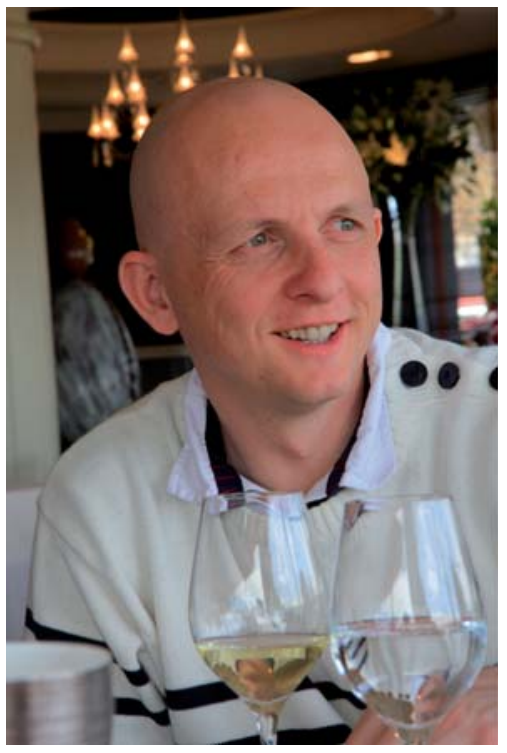

Jean-François Allemand est professeur à l'École normale supérieure. Il est ancien élève de l'École normale supérieure de Cachan, agrégé de physique, docteur de l'université Pierre et Marie Curie. Il a enseigné la biophysique en L3, en master et dans des écoles pour doctorants à des physiciens, des chimistes ou des biologistes. Il mène ses activités de recherche au sein du laboratoire de physique statistique de l'ENS sur les propriétés élastiques de l'ADN, les moteurs moléculaires travaillant sur l'ADN, in vitro et in vivo par des techniques de micromanipulations et de fluorescence. 


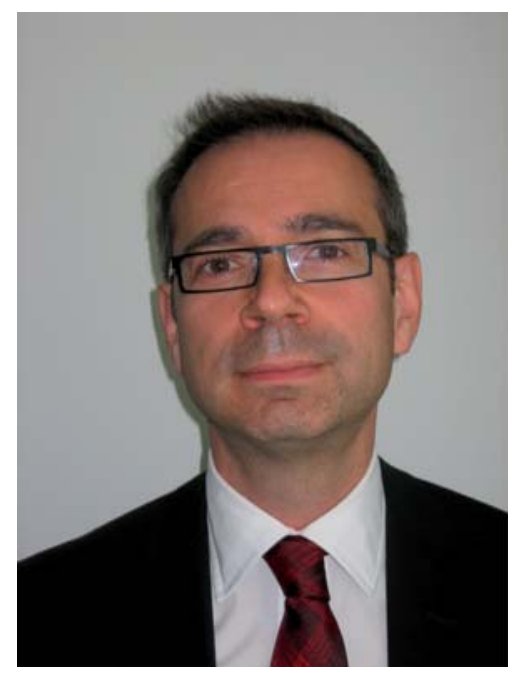

Pierre Desbiolles est inspecteur général de l'éducation nationale. Ancien élève de l'École normale supérieure, agrégé de physique, docteur en physique quantique, il a été professeur à l'université Pierre et Marie Curie. Il a mené toutes ses activités de recherche à l'École normale supérieure, au sein du laboratoire Kastler Brossel. Après avoir travaillé dans le domaine des atomes froids, en particulier sur la condensation de Bose-Einstein, il a mené des expériences utilisant la microscopie de fluorescence pour étudier les interactions entre $\mathrm{ADN}$ et protéines, à l'échelle de la molécule unique.

\section{Les contributeurs}

Olivier Bénichou, David Bensimon, Laurent Bourdieu, Vincent Croquette, Maxime Dahan, Sylvie Hénon, Jean-François Léger, Giuseppe Lia, Pascal Martin, Terence Strick, Cécile Sykes et Raphaël Voituriez ont participé avec enthousiasme à la rédaction de ce livre. 